\title{
New method of viscosity measurement near the gelatin sol-gel transition
}

\author{
J. Dumas and J.-C. Bacri \\ Laboratoire d'Ultrasons (*), Université Pierre-et-Marie-Curie, Tour 13, 4, place Jussieu, 75230 Paris Cedex 05, France
}

(Reçu le 28 janvier 1980, accepté le 28 avril 1980)

\begin{abstract}
Résumé. - La mesure d'un temps caractéristique de relaxation de rotation de petites particules ferromagnétiques dispersées dans une solution à $7 \%$ de gélatine, permet l'observation de la viscosité au voisinage de la transition sol-gel.

L'exposant critique de la viscosité $s=0,95 \pm 0,10$, valable sur 3 décades en température, est en accord avec des calculs numériques effectués dans le cadre d'une approximation de champ effectif en théorie de la percolation.

Abstract. - The viscosity of a $7 \%$ gelatin solution has been measured in the very close vicinity of the gelation transition, by rotational relaxation of ferromagnetic particles imbedded in the mixture.

The viscosity critical exponent $s=0.95 \pm 0.10$ which is obtained over 3 decades in temperature, is well accounted for by numerical calculations in the effective field treatment of percolation theory.
\end{abstract}

Gelatin is the water-soluble product of the dissolution, disorganization or degradation of waterinsoluble collagen fibers [1]. The resulting peptide chains take up random configurations and at sufficiently high temperature the mixture is a blend of monomers in a form of coils, and solvent. As the temperature is decreased, polymerization and reorganization of some helical structure occur with the formation of $n$-mers and subsequent growing of the gyration radius of the macromolecular clusters. An infinite polymer appears in the bath, at the temperature $T_{\mathrm{g}}$ of the reversible liquid-gel transition, but there are still a non-negligible amount of small $n$-mers in the system. For $T<T_{\mathrm{g}}$, the gel fraction $S_{\infty}$ - the infinite polymer - grows by linkage of the residual $n$-mers. When the gel fraction is completed by all monomers incorporation, curing of the gel occurs by the linkage of pending chains to form an unique infinite crosslinked network at $T \ll T_{\mathrm{g}}$.

The macroscopic viscosity $\bar{\eta}$ of the sol-phase depends of the size of the polymer clusters : $\bar{\eta}$ grows when the temperature is lowered to attain an infinite value when $T=T_{\mathrm{g}}$.

In this letter, we present a very simple method for observing the growing of the macroscopic viscosity in the sol-phase when $T_{\mathrm{g}}$ is approached and for measuring the corresponding critical exponent.

(*) Associated with the Centre National de la Recherche Scientifique.
1. Experimental method. - The method of measurement is based on the study of rotational relaxation time of small aggregates of magnetic particles imbedded in the gelatin solution.

In a uniform magnetic field the particles experience a torque and line up with the field : the solution shows a linear birefringence [2]. We observe the decreasing birefringence signal when the field is turned off.

We use a commercially available product, AO5 ferrofluid (from Ferrofluidic Corporation, U.S.A.) which is a colloidal suspension of monodomain ferromagnetic $\mathrm{Fe}_{3} \mathrm{O}_{4}$ particles, roughly spherically shaped, of dimension around $125 \AA$, coated with a monomolecular layer that acts as an elastic cushion preventing agglomeration.

In fact when the solution is submitted once to a small magnetic field $(\simeq 20 \mathrm{G})$, the ferrofluid particles in water-base suspension form needle-shaped agglomerates which are very difficult to destroy [3].

The size distribution of these needles is not known, but observations from electronic microscope pictures [4] suggests that the number of individual grain is very low, the main structure being composed of chains ranging from $0.1 \mu$ to more than $10 \mu$.

1.1 SAmples PREPARATION. - A gelatin solution in water was prepared with the following composition : $7 \mathrm{~g}$ of gelatin powder (from Prolabo, Paris) are dissolved in $100 \mathrm{~cm}^{3}$ of hot water and kept at $70^{\circ} \mathrm{C}$ during $15 \mathrm{~min}$. 
A drop of water-based ferrofluid is introduced in the gelatin solution to obtain a mixture with $10^{13}$ magnetic particles $/ \mathrm{cm}^{3}$. Reference solutions of the same concentration of ferrofluid in pure water have also been made to measure the size distribution of the magnetic agglomerates in a liquid of known viscosity.

The liquid mixture is introduced in a $(10 \times 3 \times 3) \mathrm{mm}$ brass tank in which optical windows allows a laser beam to go through the sample. A platinum sensor is immersed directly in the liquid to measure the temperature.

The tank is fixed on a Peltier effect temperature regulation system giving a stabilization of the tank temperature of $5 \times 10^{-3} \mathrm{~K}$.

1.2 BirefringenCE MEASUREMENTS. - Figure 1 shows the sensitive set-up used in these measurements. The principle of operation is described elsewhere [5]. The voltage obtained at the output of the lock-in detector is directly proportional to the birefringence of the sample. In our system, we cannot observe any decay rate of the birefringence signal which is more rapid than $0.5 \mathrm{~ms}$.

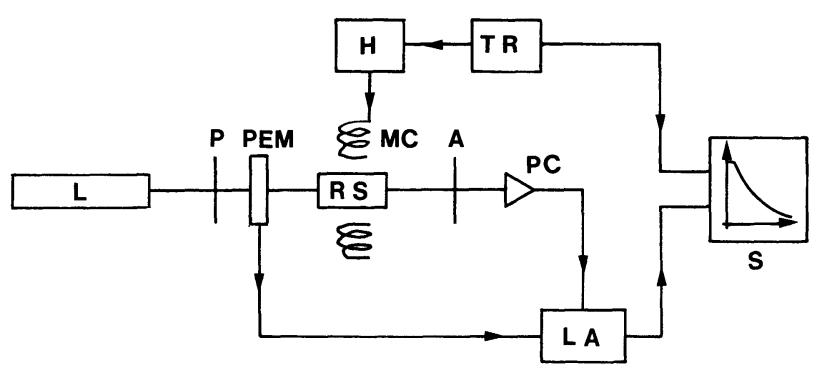

Fig. 1. - Experimental set-up. Laser L, analyser and polarizer A and $\mathrm{P}$, photoelastic phase modulator PEM, magnetic coils MC and pulsed alimentation $\mathrm{H}$, temperature regulated sample $\mathrm{RS}$, photocell PC, lock-in amplifier LA, trigger TR and scope $S$

1.3 Magnetic coils. - The sample tank is surrounded by two magnetic Helmholtz coils. The alimentation of the coils delivers adjustable current pulses of $5 \mathrm{~A}, 4 \mathrm{~s}$ long and $100 \mu \mathrm{s}$ fall time. The magnetic field available at the tank location was near $100 \mathrm{G}$. The current pulse is triggered manually or at a very slow rate $\left(10^{-2} \mathrm{~Hz}\right)$ to prevent parasitic heating of the tank.

1.4 EXPERIMENT METHOD. - The temperature of the sample is maintained stable within $10^{-3} \mathrm{~K}$ and a current pulse is applied on the magnetic coils : the birefringence signal grows, shows a constant value during the main duration of the pulse and decreases after the cut-off of the field. This decrease is recorded as a function of time on an oscilloscope and then photographed. In this preliminary experiment the analysis has been done on the photograph serie.

The measurement begins in the sol-phase of the gelatin solution just after the sample preparation, near $38^{\circ} \mathrm{C}$. Then for any temperature stabilized during the cooling, the relaxing birefringence signal is recorded. The thermodynamic equilibrium is expected to be attained in less than $5 \mathrm{~min}$. for a $1 \mathrm{~K}$ variation as can be deduced from quenching experiments on gelatin of closed composition $(6.5 \%)$ [6].

\subsection{EXPERIMENTAL RESUlts.}

- The main observed feature is the increase of the total time needed for the birefringence signal to relax $g(t)$ when the temperature decreases in the solphase : it varies in a ratio $10^{3}$ between $34^{\circ} \mathrm{C}$ and the gel transition temperature $T_{\mathrm{g}}=23.8^{\circ} \mathrm{C}$.

- The relaxing signal does not follow an exponential variation as function of time : the best fit is obtained with

$$
\frac{g\left(t_{0}\right)-g(t)}{g\left(t_{0}\right)}=\frac{1}{\log \left(\frac{t_{\mathrm{m}}}{t_{0}}\right)} \log \left(\frac{t}{t_{0}}\right)
$$

as is shown by the semi-log drawing on figure 2 . The time $t_{0}$ corresponds to the beginning of the experiment. In fact, $t_{0}$ cannot be taken smaller than $0.5 \mathrm{~ms}$ due to the bandwidth of the lock-in amplifier out-put. $t_{\mathrm{m}}$ is the time needed by $g$ to attain practically the noise level.

Figure 2 shows only the analysis of the relaxing signal near $29^{\circ} \mathrm{C}$. The logarithmic variation is observed in the whole range of temperature attained.

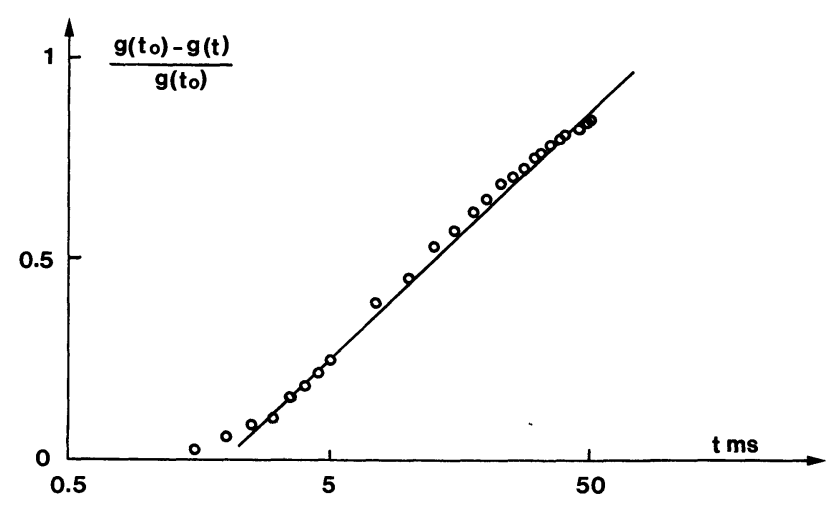

Fig. 2. - Analysis of the birefringence relaxation at $29^{\circ} \mathrm{C}$ as a function of time on a linear-log plot ;

$$
\frac{g\left(t_{0}\right)-g(t)}{g\left(t_{0}\right)}=\frac{1}{\log \left(\frac{t_{\mathrm{m}}}{t_{0}}\right)} \log \left(\frac{t}{t_{0}}\right) .
$$

The meaning of $t_{0}$ and $t_{\mathrm{m}}$ is explained in the text.

In spite of the fact that the variation is non-exponential, the time $\Delta t$ needed to divide the birefringence signal $g$ by a factor $e$, has been measured and is given versus $\left(T-T_{\mathrm{g}}\right)$ on a linear (Fig. 3) and log-log plots (Fig. 4). The experimental points are correctly 
aligned for 3 decades of variation in $\Delta T=T-T_{\mathrm{g}}$ and defined a slope (Fig. 4)

$$
s=0.95 \pm 0.10 \text {. }
$$

- The sol-gel transition is determined very sharply $\left(\simeq 10^{-2} \mathrm{~K}\right)$ as the temperature at which the birefringence signal begins decreasing as a consequence of the locking of the ferrofluid chains by the infinite network. The absolute value of $s$, measured over $10 \mathrm{~K}$, is practically not affected by this uncertainty.

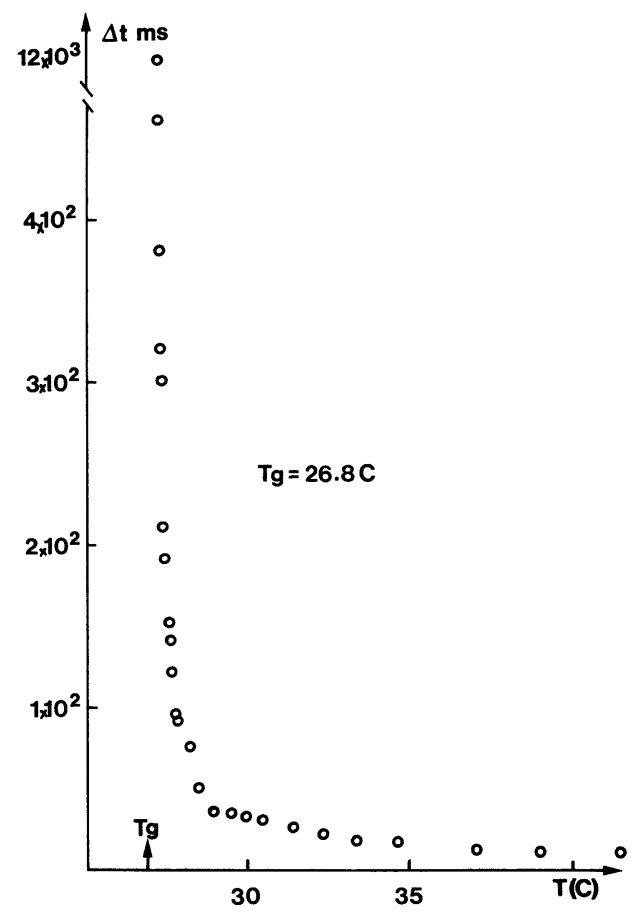

Fig. 3. - Characteristic time $\Delta t$ of birefringence relaxation as a function of the temperature. It is shown in the text that $\Delta t$ is proportional to the macroscopic viscosity $\bar{\eta}$.

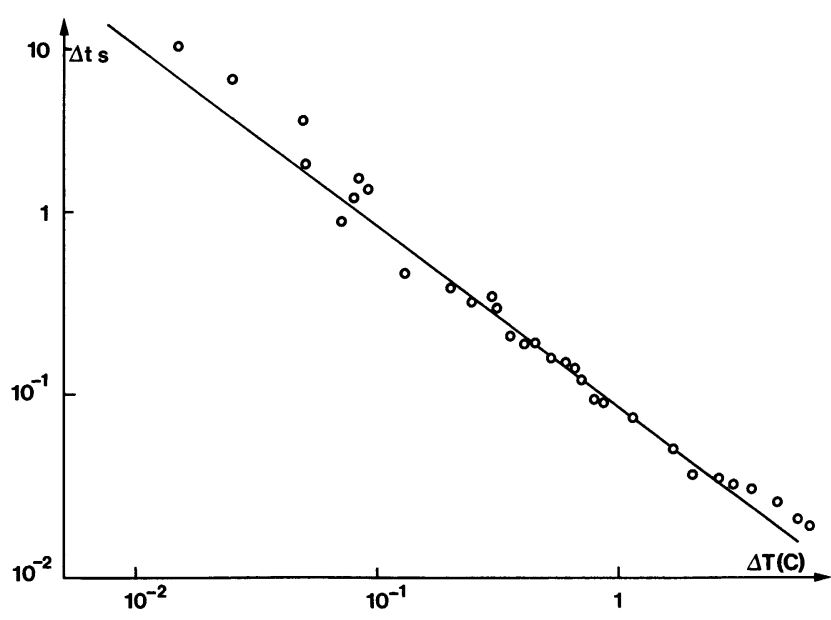

Fig. 4. - Log-log plot of the characteristic time $\Delta t$ of the birefringence as a function of the temperature difference $\Delta T=T-T_{\mathrm{g}}$ where $T_{\mathrm{g}}=26.8^{\circ} \mathrm{C}$, is the gelation point. The slope $s=0.95 \pm 0.10$ is the critical exponent of the macroscopic viscosity $\bar{\eta}$.
2. Theory. - The relaxing birefringence signal $g(t)$ is proportional to the number of optically anisotropic ferrofluid particles :

$$
g(t)=G \int_{1}^{F} f \varphi_{f} \mathrm{e}^{-D_{f} t} \mathrm{~d} f
$$

where $G$ is a constant depending on the birefringence of all the particles, $D_{f}$ the rotation diffusion constant of a chain of $f$ magnetic particles, and $\varphi_{f}$ the number concentration of chains formed by $f$ particles. $F$ is the number of magnetic grains belonging to the longest chain.

In the case of long prolate ellipsoids the rotationdiffusion constant $D_{f}$ is related mainly to the length $a$ of the elongated particles and shows very little dependence on any other dimensions $b$ of the ellipsoid [7] :

$$
D_{f}=\frac{k T}{16 \pi \eta a^{3}} 3\left[2 \ln \left(\frac{2 a}{b}\right)-1\right] \text {. }
$$

We then take the coefficient $D_{f}$ corresponding to an equivalent sphere of radius $r_{f} \sim a$ which takes into account all important parameters :

$$
D_{f} \simeq \frac{k T}{8 \pi \eta r_{f}^{3}} .
$$

We suppose $r_{f}=a_{1} f^{\delta}$ where $\delta$ is an unknown parameter and $a_{1}$ the ferrofluid one-particle radius.

The most important terms in the integral correspond to values of $f$ and $t$ for which the exponential argument :

$$
D_{f} t \leqslant 1
$$

this expression defining the lower limit of integration.

$$
f^{*}=\left(\frac{k T}{8 \pi a_{1}^{3}} \frac{t}{\eta}\right)^{1 / 3 \delta} .
$$

We expect that for a given value of $t / \eta$ the birefringence signal is mainly due to particles ranging from $f^{*}$ to $F$. A rough calculation of $r_{f^{*}}$, taking the exact value of $D_{f}$, with $a / b \sim 50$ and $\delta=1$, gives a value of $0.1 \mu$ : it is probable that all birefringent particles are taken into account in this approximation.

As is pointed in [8] the value of the viscosity measured depends on the probe dimension $r_{f}$, and the macroscopic viscosity $\bar{\eta}$ is obtained only for $r_{f} \geqslant \xi=r_{N^{*}}$, with $\xi$ the characteristic length of the largest clusters of gelatine : with a monomer coil radius $100 \AA$, the correlation length of $10 \mu$ is obtained for $\Delta p \simeq 10^{-3}$. Thus a viscosity correction should have perhaps been made for the last decade in our measurement.

The experimental variation $g(t)$ is logarithmic (Fig. 2) and this suggests that the size distribution $\varphi_{f}$ of the ferrofluid chains is :

$$
\varphi_{f} \simeq \frac{1}{f^{2}} .
$$


Then, the birefringence relaxing signal is :

$$
g(t) \simeq G\left[\log F-\frac{1}{3 \delta} \log \left(\frac{k T}{6 \pi a_{1}^{3}} \frac{t}{\eta}\right)\right] .
$$

We measure a certain interval of time $\Delta t$ on the relaxing signal defined by :

$$
\frac{1}{e} g\left(t_{0}\right)=g\left(t_{0}+\Delta t\right) .
$$

In our case we take $t_{0} \simeq 0.5 \mathrm{~ms}$ and, as $\Delta t$ varies from $8 \mathrm{~ms}$ to $12 \mathrm{~s}, t_{0} \ll t$ is a very good approximation. In these conditions, we find that $\Delta t$ is proportional to the macroscopic viscosity $\bar{\eta}: \bar{\eta} \sim \Delta t$ noticing that $t_{0} / \eta$ has a constant value, which is a consequence of the experimentally observed constant level of $g\left(t_{0}\right)$. The viscosity critical exponent $s$ is defined by :

$$
\bar{\eta}=\eta_{0}|\Delta p|^{-s}
$$

in which $\Delta p=p-p_{\mathrm{c}}$, where $p$ is the fraction of reacted bonds and $p_{\mathrm{c}}$ the gelation threshold. $\eta_{0}$ is the viscosity of the original solution of monomers at high temperature. Supposing a linear relation between $\Delta p$ and $\Delta T=T-T_{\mathrm{g}}$ in the vicinity of $T_{\mathrm{g}}$, the slope of the log-log drawing of $\Delta t(T)$ (Fig. 4) gives the critical exponent $s$.

It can be remarked that the result $\Delta t \sim \bar{\eta}$ would have been found for any distribution $\varphi_{F} \simeq 1 / f^{\varepsilon}, \varepsilon>1$ : this approximation is not critical in the calculation.

3. Discussion. - In spite of the fact that the classical theory of gelation [9] does not attempt to predict the viscosity behaviour, three values of the critical exponent $s$ are available in the literature [10, $11,12]$. Among them, the effective medium approximation of the percolation theory [12] gives a value $s=1$, which is very well verified in our experiment. This exponent has already been found experimentally in styrene divinylbenzene gelating system [13] and governs the viscosity behaviour far from the transition point, whereas $s \simeq 0.78$ very close to the transition; this last value has been numerically calculated and found for the electrical conductance in random mixtures of normal and superconducting elements [11] which as certain arguments suggest [10] follow the same behaviour as the gelating system viscosity.

We do not find any indications of such a crossover in the gelatin system.

Some remarks can be made at this stage.

- The effective medium approximation is a mean field treatment and applies only outside a critical region, which is difficult to appreciate in the gelatin case as the connection is not known between $p$ and $T$, in spite of certain theoretical arguments [14]. We can only claim that $\Delta p$ is proportional to $\Delta T$ in the immediate vicinity of $T_{\mathrm{g}}$, and this approximation is used here.

- The calculations and theories that we just mentioned are concerned with chemical gelation which supposes strong and not reversible crosslinking between polymers. It is not obvious that the same kind of theoretical treatment can be applied to the gelatin system.

Aknowledgments. - The authors thank P. G. de Gennes and F. Brochard for a helpful and stimulating discussion.

\section{References}

[1] VeIS, A., The macromolecular chemistry of gelatin (Academic Press) 1964.

[2] Martinet, A., Rheol. Acta 13 (1974) 260.

[3] Hayes, C. F., Hwang, S. R., J. Colloid Interface Sci. 60 (1977) 443

[4] Horn Chandesris, D., Thèse de 3e cycle, Orsay (mars 1977).

[5] Kemp, J. C., J. Opt. Soc. Am. 59 (1969) 950.

[6] Eagland, D., Pilling, G. and Wheeler, R. G., Farad. Discuss. Chem. Soc. 57 (1974) 181.

[7] TANFord, C., Physical Chemistry of Macromolecules (John Wiley and Sons) 1965.
[8] De Gennes, P. G., J. Physique Lett. 40 (1979) L-197.

[9] FloRY, P. J., Principles of polymer chemistry (Cornell Univ. Press, Ithaca) 1953.

[10] De Gennes, P. G., C.R. Hebd. Séan. Acad. Sci. Paris 286B (1978) 131

[11] Straley, J. P., J. Phys. C (Sol. St. Phys.) 9 (1976) 783.

[12] Kirkpatrick, S., Rev. Mod. Phys. 45 (1973) 574.

[13] Adam, M., Delsanti, M., Okasha, R., Hild, G., J. Physique Lett. 40 (1979) L-539.

[14] Kastekeyn, P. W., Fortuin, C. M., J. Phys. Soc. Japan 26 Suppl. (1969) 11. 